\title{
Clinical Pharmacogenetics Implementation Consortium Guidelines for HLA-B Genotype and Abacavir Dosing: 2014 Update
}

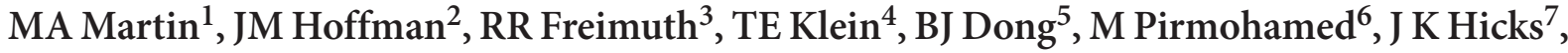 \\ MR Wilkinson ${ }^{2}$, DW Haas ${ }^{8}$ and DL Kroetz ${ }^{1}$
}

The Clinical Pharmacogenetics Implementation Consortium (CPIC) Guidelines for $H L A-B$ Genotype and Abacavir Dosing were originally published in April 2012. We reviewed recent literature and concluded that none of the evidence would change the therapeutic recommendations in the original guideline; therefore, the original publication remains clinically current. However, we have updated the Supplementary Material online and included additional resources for applying CPIC guidelines to the electronic health record. Up-to-date information can be found at PharmGKB (http://www.pharmgkb.org).

The Clinical Pharmacogenetics Implementation Consortium (CPIC) of the Pharmacogenetics Research Network (http:// www.pgrn.org) and the Pharmacogenomics Knowledge Base (PharmGKB, http://www.pharmgkb.org) provide peer-reviewed, evidence-based, freely accessible, genotype-based drug guidelines to help clinicians understand how available genetic test results could be used to optimize drug therapy. ${ }^{1}$ CPIC guidelines undergo continuous peer review, and information that would modify prescribing recommendations pertaining to gene-specific alleles and nomenclature is updated periodically on the PharmGKB website. Furthermore, published CPIC guidelines are currently systematically reviewed for updates every 2-3 years.

The Clinical Pharmacogenetics Implementation Consortium Guidelines for $H L A-B$ Genotype and Abacavir Dosing were originally published in April 2012. ${ }^{2}$ To update this guideline, we conducted a focused review of the literature published between April 2011 and November 2013 on HLA-B genotype and abacavir use (see Supplementary Material online). The minor allele frequency table based on ethnicity is already extensive and has not been updated at this time (see Supplementary Tables S1 and S2 online). The literature review yielded one additional study that we added to the evidence table in the supplementary material $^{3}$ (see Supplementary Table S3 online). We found no new evidence that would change our original recommendations; therefore, the original guideline publication remains current. ${ }^{2}$ However, recent studies have been published that further describe the mechanism by which abacavir can elicit an immune response through $H L A-B^{\star} 57: 01$. Discussion of these studies was added to the Supplementary Material online. Supplementary Figures S1 and S2 online also remain unchanged.

Recently, the CPIC Steering Committee recommended that authors address dosing in pediatrics in all updated and new CPIC guidelines. Although much of the evidence linking HLA$B^{\star} 57: 01$ to abacavir hypersensitivity was conducted in adults, there is no reason to suspect that children positive for HLA$B \star 57: 01$ would be at less risk for abacavir hypersensitivity reactions than adults positive for $H L A-B^{\star} 57: 01$. Furthermore, the Panel on Antiretroviral Therapy and Medical Management of HIV-Infected Children (Guidelines for the Use of Antiretroviral Agents in Pediatric HIV Infection; http://aidsinfo.nih.gov/contentfiles/lvguidelines/pediatricguidelines.pdf) recommends against the use of abacavir in children who test positive for HLA-B ${ }^{\star 57: 01 .}$.

CPIC guidelines are designed to help clinicians use genetic information to optimize drug therapy; to do this effectively, pharmacogenetic information must be incorporated into electronic health records (EHRs) with clinical decision support. ${ }^{4-6}$ To provide additional resources for applying CPIC guidelines to the EHR, in 2013, CPIC created an informatics working group

\footnotetext{
${ }^{1}$ Department of Bioengineering and Therapeutic Sciences, University of California, San Francisco, San Francisco, California, USA; ${ }^{2}$ Department of Pharmaceutical Sciences, St. Jude Children's Research Hospital, Memphis, Tennessee, USA; ${ }^{3}$ Department of Health Sciences Research, Mayo Clinic, Rochester, Minnesota, USA; ${ }^{4}$ Department of Genetics, Stanford University, Stanford, California, USA; ${ }^{5}$ Department of Clinical Pharmacy, University of California, San Francisco, San Francisco, California, USA; ${ }^{6}$ Department of Pharmacology, University of Liverpool, Liverpool, UK; ${ }^{7}$ Department of Pharmacy and Center for Personalized Healthcare, Cleveland Clinic, Cleveland, Ohio, USA; ${ }^{8}$ Department of Medicine, Vanderbilt University, Nashville, Tennessee, USA. Correspondence: DL Kroetz (deanna.kroetz@ucsf.edu)
} 
focused on supporting the adoption of CPIC guidelines within a clinical electronic environment. This guideline is the first to provide these new clinical implementation resources as part of the Supplementary Material online, which includes workflow diagrams that illustrate the storage of a pharmacogenetic result in an EHR and the design of clinical decision support alerts (see Supplementary Figures S3 and S4 online). New tables that correspond to these workflow diagrams are provided that translate genotype test results into an interpreted phenotype (see Supplementary Tables S4-S8 online). These tables provide a summary of genotype/phenotype terms, example text for documentation in the EHR and point-of-care alerts, and crossreferences for drug and gene names to widely used terminologies and standardized nomenclature systems.

We recognize that each organization and EHR vendor may have different requirements and preferences for implementing pharmacogenetics within a given electronic environment. The intent of these resources is to synthesize foundational knowledge that provides a common starting point for clinical implementation so that individual organizations do not have to create a similar knowledge base for each new gene-drug pair for which pharmacogenetics is implemented. Future CPIC guidelines and guideline updates will provide similar resources to guide the implementation of gene-drug pairs in the EHR with clinical decision support.

SUPPLEMENTARY MATERIAL is linked to the online version of the paper at http://www.nature.com/cpt

\section{ACKNOWLEDGMENTS}

We acknowledge the critical input of members of the Clinical Pharmacogenetics Implementation Consortium (CPIC) of the Pharmacogenomics Research Network, particularly Mary V. Relling and Kelly E. Caudle (St. Jude Children's Research Hospital), and the CPIC Informatics Working Group, particularly Michelle Whirl-Carrillo (Stanford University) and Marc S. Williams (Geisinger Health System). This work was funded by grants from the National Institutes of Health/National Institute of General Medical Science (NIH/NIGMS) (PAAR4Kids (UO1 GM92666) and PharmGKB (R24 GM61374)).
This work was also supported by NIH U01 GM061390 (D.L.K), NIHT32 GM007175 (M.A.M.), NIH/NIGMS U19 GM61388 (R.R.F.), and NIH RO1 Al-077505 (D.W.H.).

CPIC guidelines reflect expert consensus based on clinical evidence and peer-reviewed literature available at the time they are written and are intended only to assist clinicians in decision making and to identify questions for further research. New evidence may have emerged since the time a guideline was submitted for publication. Guidelines are limited in scope and are not applicable to interventions or diseases not specifically identified. Guidelines do not account for all individual variations among patients and cannot be considered inclusive of all proper methods of care or exclusive of other treatments. It remains the responsibility of the healthcare provider to determine the best course of treatment for the patient. Adherence to any guideline is voluntary, with the ultimate determination regarding its application to be solely made by the clinician and the patient. CPIC assumes no responsibility for any injury to persons or damage to property related to any use of CPIC's guidelines or for any errors or omissions.

\section{CONFLICT OF INTEREST}

T.E.K. is a consultant for Personalis. D.W.H. has been a consultant to Merck. The other authors declared no conflict of interest.

@ 2014 American Society for Clinical Pharmacology and Therapeutics

1. Relling, M.V. \& Klein, T.E. CPIC: Clinical Pharmacogenetics Implementation Consortium of the Pharmacogenomics Research Network. Clin. Pharmacol. Ther. 89, 464-467 (2011).

2. Martin, M.A., Klein, T.E., Dong, B.J., Pirmohamed, M., Haas, D.W. \& Kroetz, D.L. Clinical Pharmacogenetics Implementation Consortium. Clinical pharmacogenetics implementation consortium guidelines for $H L A-B$ genotype and abacavir dosing. Clin. Pharmacol. Ther. 91, 734-738 (2012).

3. Schnyder, B., Adam, J., Rauch, A., Thurnheer, M.C. \& Pichler, W.J. HLA-B*57:01(+) abacavir-naive individuals have specific T cells but no patch test reactivity. J. Allergy Clin. Immunol. 132, 756-758 (2013).

4. Shuldiner, A.R. et al.; Pharmacogenomics Research Network Translational Pharmacogenetics Program Group. The Pharmacogenomics Research Network Translational Pharmacogenetics Program: overcoming challenges of real-world implementation. Clin. Pharmacol. Ther. 94, 207-210 (2013).

5. Wilke, R.A. et al. The emerging role of electronic medical records in pharmacogenomics. Clin. Pharmacol. Ther. 89, 379-386 (2011).

6. Kullo, I.J., Jarvik, G.P., Manolio, T.A., Williams, M.S. \& Roden, D.M. Leveraging the electronic health record to implement genomic medicine. Genet. Med. 15 , 270-271 (2013). 